\title{
Rotated, Scaled, and Noisy 2D and 3D Texture Classification with the Bispectrum-Based Invariant Feature
}

\author{
Yo Horikawa \\ Faculty of Engineering, Kagawa University \\ Takamatsu 761-0396, Japan \\ horikawa@eng.kagawa-u.ac.jp
}

\begin{abstract}
The author presents a novel feature of 2D and 3D images invariant to similarity transformations and robust to noise on the basis of the bispectrum. The invariant feature is applied to the classification of texture images suffering from rotation, scaling and noise. Computer experiment shows that about $90 \%$ correct classification ratio is obtained for 5 kinds of 2D natural textures and of 3D brain images rotated in arbitrary degree, scaled up to double and with the white Gaussian noise of $0 \mathrm{~dB}$ SNR. The feature can also be used to the estimation of the rotation angles of texture images.
\end{abstract}

\section{Introduction}

Texture classification invariant to geometric transformations is of importance in many practical applications [1]. Several methods for the classification invariant to the rotation and scaling of images have been proposed [2]-[15]. However, the robustness to noise has not been considered except for [8], [12]. On the other hand, the thirdorder correlation and the bispectrum are robust to the additive noise of any symmetrical distribution [16], [17]. Some methods based on the third-order statistics have been applied to noisy texture classification [18], [19] and texture synthesis [20]. The results on the classification of texture images under rotation, scaling and noise using the third-order statistics have not been obtained, however, as far as the author knows.

In this study the author presents a bispectrum-based feature of $2 \mathrm{D}$ and 3D patterns invariant to similarity transformations and robust to noise. The invariant feature is then applied to the classification of 2D and 3D texture images suffering from rotation, scaling and additive noise. It is also shown that the invariant feature is applicable to the estimation of the rotation angles of texture images.

\section{Invariant Feature Based on the Bispectrum}

The derivation of the invariant feature from the bispectrum and the effective calculation method are as follows. 
Let $f(\boldsymbol{x})$ be $2 \mathrm{D}$ or 3D image data and $F(\omega)$ be its Fourier transform.

$$
F(\omega)=\int f(x) \exp (-\mathrm{j} \omega \cdot \boldsymbol{x}) \mathrm{d} \boldsymbol{x}
$$

The bispectrum $B\left(\omega_{1}, \omega_{2}\right)$ of $f(x)$ is defined by the triple product of $F(\omega)$

$$
B\left(\omega_{1}, \omega_{2}\right)=F\left(\omega_{1}\right) F\left(\omega_{2}\right) F\left(-\omega_{1}-\omega_{2}\right)
$$

which is invariant to the shift of image data.

When image data is rotated at an arbitrary point, the bispectrum is rotated at the origin by the same angle. When image data is scaled, the bispectrum is scaled at the origin by the inversely proportional amount. We then integrate the bispectrum in the $\left(\omega_{1}, \omega_{2}\right)$ space on condition that $\left|\omega_{1}\right| /\left|\omega_{2}\right|=r, \omega_{1} \cdot \omega_{2} /\left(\left|\omega_{1}\right|\left|\omega_{2}\right|\right)=\cos \theta$.

$$
I^{\prime}(r, \theta)=\iint_{r, \theta} B\left(\omega_{1}, \omega_{2}\right) \mathrm{d} \omega_{1} \mathrm{~d} \omega_{2} \quad\left(\left|\omega_{1}\right| /\left|\omega_{2}\right|=r, \omega_{1} \cdot \omega_{2} /\left(\left|\omega_{1}\right|\left|\omega_{2}\right|\right)=\cos \theta\right)
$$

This two-dimensional function $I^{\prime}(r, \theta)$ on the $(r, \theta)$ plane represents the amount of the sinusoids of frequency components $\omega_{1}, \omega_{2}$ and $\omega_{1}+\omega_{2}$ which have the same ratio $r$ of length and the same angle $\theta$ in image data.

A feature $I(r, \theta)$ of image data invariant to similarity transformations (shift, rotation and scaling) is obtained through normalization.

$$
I(r, \theta)=I^{\prime}(r, \theta) /\left(\iint I^{, 2}(r, \theta)\right)^{1 / 2}
$$

Note that this feature is also invariant to linear changes in the gray-scale values of image data.

To avoid computational complexity in calculation and interpolation of the bispectrum of high-dimensional (2D or 3D) data, a simple and effective method of calculating the invariant feature is introduced.

Let $f(\boldsymbol{x}) \quad(\boldsymbol{x}=(x, y), x, y=0, \cdots, N-1)$ be $2 \mathrm{D}$ digital image data of $N \times N$ pixels. (Extension to 3D images is straightforward.) The Fourier transform $F(\omega)$ of image data is calculated with $2 N \times 2 N$ point FFT using the Gaussian window $\exp (-(\boldsymbol{x}-\mu) \cdot(\boldsymbol{x}$ $\left.\mu) /\left(2 \sigma^{2}\right)\right) \quad(\mu=(N / 2, N / 2), \sigma=N / 3)$ and padding zero outside of $N \times N$ data. For each frequency pair $\left(\omega_{1}, \omega_{2}\right) \quad\left(\omega_{I}=\left(\omega_{x}, \omega_{y}\right)-N / 2 \leq \omega_{x}, \omega_{y}<N / 2\right.$ (integer)), the triple product $F\left(\omega_{1}\right) F\left(\omega_{2}\right) F\left(-\omega_{1}-\omega_{2}\right)$ is calculated. (The value of $F\left(-\omega_{1}-\omega_{2}\right.$ : non-integer) is calculated with the bilinear interpolation.) Its value is then added up into appropriately divided classes of $(r, \theta)$, where $\left|\omega_{1}\right| /\left|\omega_{2}\right|=r$ and $\left.\omega_{1} \cdot \omega_{2} /\left(\left|\omega_{1}\right|\left|\omega_{2}\right|\right)=\cos \theta\right)$. The obtained table (histogram) on $(r, \theta)$ corresponds to $I^{\prime}(r, \theta)$ and the normalization leads to an estimate of the invariant feature $I(r, \theta)$. The calculation is done in $O\left(N^{4}\right)$ time and in $O\left(N^{2}\right)$ space. (For $d$-dimensional data, the computational time is in $O\left(N^{2 d}\right)$ and the space is in $O\left(N^{d}\right)$, where $N^{d}$ is the number of pixels of image data.)

\section{Texture Classification Experiments}

The invariant feature is applied to the classification of 2D natural texture images and 3D brain images under rotation, scaling and additive noise. 


\subsection{D Natural Textures}

Five natural texture images: (a) D4 (Pressed cork), (b) D12 (Bark of tree), (c) D15 (Straw), (d) D17 (Herringbone weave) and (e) D84 (Raffia looped to a high pile) are taken from the Brodatz album [21]. The pictures of the textures are digitized to 8 bits gray-scaled image data of $260 \times 240$ pixels and their central parts of $128 \times 128$ pixels are used in the experiment.

Figure 1 shows the invariant features $I(r, \theta)$, where $\operatorname{Re}(I(r, \theta))$ is plotted since $\operatorname{Im}(I(r, \theta)) \approx 0$ owing to $F(-\omega)=-F(\omega)$. The ratio $r$ is ranged from 1.0 to 10 by $10^{0.2}$ and the interior angle $\theta$ is ranged from $0^{\circ}$ to $180^{\circ}$ by $18^{\circ}$. The graphes of the invariant features have different shapes.

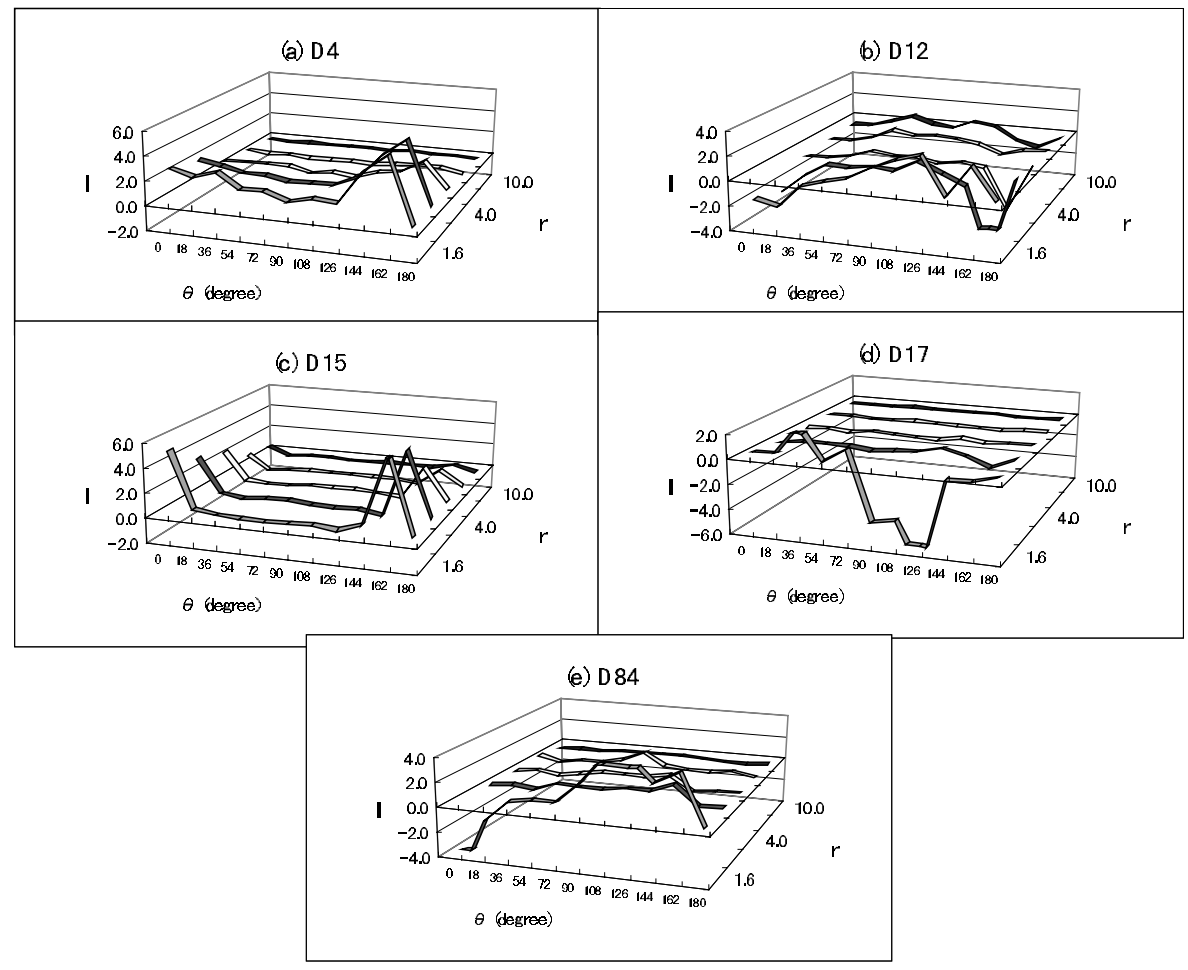

Fig. 1. Invariant feature $I(r, \theta)$ of $2 \mathrm{D}$ textures.

Table 1 shows the RMS distances between the features of the original images and those of transformed and noisy images. The transformed images are made with the bilinear interpolation of the original images. For three scaling factors $(1.0,1.5,2.0)$, the images are shifted from 0 to 0.5 by 0.1 and are rotated from $0^{\circ}$ to $45^{\circ}$ by $5^{\circ}$. A hundred noisy images are made by adding the white Gaussian noise $\mathrm{N}\left(0,40^{2}\right)$ (SNR is about $0 \mathrm{~dB}$ ) to the original images. All the transformed and noisy images are correctly classified with the RMS distance to the invariant features of the original images. 
Table 1. RMS distance of the invariant feature of transformed 2D texture images.

\begin{tabular}{|c|c|c|c|c|c|c|}
\hline & & (a) & (b) & (c) & (d) & (e) \\
\hline & & $\begin{array}{l}\text { Max. Mean } \\
\text { Min. }\end{array}$ & $\begin{array}{l}\text { Max. Mean } \\
\text { Min. }\end{array}$ & $\begin{array}{l}\text { Max. Mean } \\
\text { Min. }\end{array}$ & $\begin{array}{l}\text { Max. Mean } \\
\text { Min. }\end{array}$ & $\begin{array}{l}\text { Max. Mean } \\
\text { Min. }\end{array}$ \\
\hline & $x 1.0$ & $\begin{array}{cc}0.23 & 0.16 \\
0.0 & \end{array}$ & $\begin{array}{cc}1.73 & 1.70 \\
1.67 & \end{array}$ & $\begin{array}{cc}0.98 & 0.93 \\
0.86\end{array}$ & $\begin{array}{l}1.50 \\
1.39\end{array}$ & $\begin{array}{cc}1.25 & 1.22 \\
1.19 & \end{array}$ \\
\hline & $x 1.0$ & $\begin{array}{cc}1.74 & 1.68 \\
1.60 & \end{array}$ & $\begin{array}{cc}0.57 & 0.37 \\
0.0 & \\
\end{array}$ & $\begin{array}{cc}1.77 & 1.69 \\
1.59 & \\
\end{array}$ & $\begin{array}{l}1.22 \\
1.09\end{array}$ & $\begin{array}{c}1.54 \\
1.43\end{array} 1.49$ \\
\hline (c) & $x 1.0$ & $\begin{array}{c}1.00 \\
0.89\end{array}$ & $\begin{array}{cc}1.80 & 1.76 \\
1.72 & \end{array}$ & $\begin{array}{cc}0.51 & 0.34 \\
0.0 & \end{array}$ & $\begin{array}{c}1.44 \\
1.38\end{array}$ & $\begin{array}{cc}1.53 & 1.43 \\
1.31 & \end{array}$ \\
\hline (d) & $x 1.0$ & $\begin{array}{c}1.44 \\
1.39\end{array}$ & $\begin{array}{c}1.12 \\
1.07\end{array}$ & $\begin{array}{cc}1.45 & 1.44 \\
1.41 & \end{array}$ & $\begin{array}{cc}0.23 & 0.17 \\
0.0 & \\
\end{array}$ & $\begin{array}{cc}1.73 & 1.72 \\
1.71 & \\
\end{array}$ \\
\hline (e) & $x 1.0$ & $\begin{array}{cc}1.33 & 1.22 \\
1.16 & \\
\end{array}$ & $\begin{array}{cc}1.53 & 1.47 \\
1.37 & \end{array}$ & $\begin{array}{cc}1.46 & 1.38 \\
1.33 & \end{array}$ & $\begin{array}{ll}1.76 & 1.72 \\
1.68 & \\
\end{array}$ & $\begin{array}{cc}0.51 & 0.30 \\
0.0 & \end{array}$ \\
\hline & $\mathrm{X} 1.5$ & $\begin{array}{ll}0.31 & 0.27 \\
0.21\end{array}$ & $\begin{array}{cc}1.73 & 1.71 \\
1.68 & \\
\end{array}$ & $\begin{array}{cc}0.89 & 0.83 \\
0.78\end{array}$ & $\begin{array}{cc}1.54 & 1.49 \\
1.45 & \end{array}$ & $\begin{array}{ll}1.20 & 1.16 \\
1.11 & \end{array}$ \\
\hline & $x 1.5$ & $\begin{array}{cc}1.80 & 1.76 \\
1.72 & \end{array}$ & $\begin{array}{cc}0.65 & 0.62 \\
0.57 & \end{array}$ & $\begin{array}{cc}1.84 & 1.74 \\
1.65 & \end{array}$ & $\begin{array}{cc}0.98 & 0.92 \\
0.87 & \end{array}$ & $\begin{array}{c}1.61 \\
1.44\end{array}$ \\
\hline (c) & $x 1.5$ & $\begin{array}{cc}0.91 & 0.85 \\
0.81 & \end{array}$ & $\begin{array}{ll}1.81 & 1.78 \\
1.76 & \end{array}$ & $\begin{array}{cc}0.68 & 0.49 \\
0.44 & \end{array}$ & $\begin{array}{c}1.47 \\
1.39\end{array}$ & $\begin{array}{c}1.58 \\
1.42\end{array}$ \\
\hline (d) & $\mathrm{X} 1.5$ & $\begin{array}{c}1.48 \quad 1.42 \\
1.35\end{array}$ & $\begin{array}{ll}1.15 & 1.09 \\
1.05 & \end{array}$ & $\begin{array}{c}1.43 \\
1.34\end{array}$ & $\begin{array}{l}0.320 .31 \\
0.29\end{array}$ & $\begin{array}{ll}1.75 & 1.72 \\
1.70 & \\
\end{array}$ \\
\hline (e) & $x 1.5$ & $\begin{array}{cc}1.39 & 1.34 \\
1.24 & \\
\end{array}$ & $\begin{array}{cc}1.46 & 1.41 \\
1.39 & \\
\end{array}$ & $\begin{array}{c}1.45 \\
1.34\end{array}$ & $\begin{array}{ll}1.65 & 1.63 \\
1.60 & \\
\end{array}$ & $\begin{array}{cc}0.64 & 0.59 \\
0.49 & \end{array}$ \\
\hline & $\times 2.0$ & $\begin{array}{ll}0.52 & 0.38 \\
0.28 & \\
\end{array}$ & $\begin{array}{cc}1.74 & 1.68 \\
1.62 & \\
\end{array}$ & $\begin{array}{cc}0.86 & 0.83 \\
0.77\end{array}$ & $\begin{array}{c}1.51 \\
1.40\end{array}$ & $\begin{array}{c}1.24 \\
1.07\end{array}$ \\
\hline (b) & $\times 2.0$ & $\begin{array}{cc}1.93 & 1.85 \\
1.80 & \end{array}$ & $\begin{array}{cc}1.02 & 0.96 \\
0.86 & \end{array}$ & $\begin{array}{cc}1.85 & 1.74 \\
1.56 & \\
\end{array}$ & $\begin{array}{c}1.181 .06 \\
0.96\end{array}$ & $\begin{array}{l}\text { 1. } 72 \\
\text { 1. } 46\end{array}$ \\
\hline (c) & $\times 2.0$ & $\begin{array}{cc}0.89 & 0.78 \\
0.66\end{array}$ & $\begin{array}{cc}1.81 & 1.78 \\
1.75 & \end{array}$ & $\begin{array}{cc}0.64 & 0.51 \\
0.44 & \\
\end{array}$ & $\begin{array}{cc}1.49 & 1.46 \\
1.41 & \\
\end{array}$ & $\begin{array}{c}1.46 \\
1.36\end{array}$ \\
\hline (d) & $\times 2.0$ & $\begin{array}{l}1.47 \\
1.42\end{array}$ & $\begin{array}{c}1.17 \\
1.00\end{array}$ & $\begin{array}{cc}1.48 & 1.38 \\
1.30 & \end{array}$ & $\begin{array}{cc}0.49 & 0.42 \\
0.38\end{array}$ & $\begin{array}{c}1.83 \\
1.70\end{array}{ }^{1.76}$ \\
\hline$(e)$ & $\times 2.0$ & $\begin{array}{cc}1.55 & 1.28 \\
1.04 & \end{array}$ & $\begin{array}{cc}1.53 & 1.46 \\
1.39 & \end{array}$ & $\begin{array}{l}1.651 .36 \\
1.04\end{array}$ & $\begin{array}{cc}1.77 & 1.52 \\
1.36 & \end{array}$ & $\begin{array}{c}1.00 \\
0.74\end{array}$ \\
\hline & Noise & $\begin{array}{l}0.250 .17 \\
0.10^{0}\end{array}$ & $\begin{array}{cc}1.78 & 1.71 \\
1.62 & \\
\end{array}$ & $\begin{array}{c}0.970 .90 \\
0.80\end{array}$ & $\begin{array}{l}1.561 .43 \\
\text { 1. } 29\end{array}$ & $\begin{array}{c}1.35 \\
1.12\end{array}$ \\
\hline & Noise & $\begin{array}{cc}1.78 & 1.71 \\
1.64 & \end{array}$ & $\begin{array}{cc}0.33 & 0.22 \\
0.13\end{array}$ & $\begin{array}{cc}1.83 & 1.76 \\
1.68 & \\
\end{array}$ & $\begin{array}{c}1.83 \\
1.68\end{array}$ & $\begin{array}{c}1.59 \\
1.40\end{array}$ \\
\hline & Noise & $\begin{array}{cc}0.98 & 0.89 \\
0.83\end{array}$ & $\begin{array}{c}1.81 \quad 1.76 \\
1.73\end{array}$ & $\begin{array}{l}0.24 \quad 0.15 \\
0.09\end{array}$ & $\begin{array}{c}1.49 \\
1.38\end{array}$ & $\begin{array}{c}1.48 \quad 1.40 \\
1.31\end{array}$ \\
\hline (d) & Noise & $\begin{array}{c}1.50 \\
1.37\end{array}$ & $\begin{array}{c}1.15 \\
1.03\end{array}$ & $\begin{array}{cc}1.50 & 1.44 \\
1.39 & \end{array}$ & $\begin{array}{ll}0.23 & 0.14 \\
0.08\end{array}$ & $\begin{array}{cc}1.77 & 1.73 \\
1.66 & \end{array}$ \\
\hline (e) & Noise & $\begin{array}{cc}1.35 & 1.26 \\
1.11 & \\
\end{array}$ & $\begin{array}{cc}1.59 & 1.51 \\
1.40 & \\
\end{array}$ & $\begin{array}{cc}1.51 & 1.40 \\
1.26 & \\
\end{array}$ & $\begin{array}{cc}1.80 & 1.72 \\
1.62 & \\
\end{array}$ & $\begin{array}{ll}0.37 & 0.21 \\
0.11 & \\
\end{array}$ \\
\hline
\end{tabular}

Next, classification experiment on image data with random transformations and additive noise is done. The original image data are randomly shifted in [-40.0, 40.0], rotated in $\left[0^{\circ}, 360^{\circ}\right]$ and scaled by the factor in $[1.0,2.0]$. The white Gaussian noise with the standard deviation (S.D.) 40,80 and 120 (SNR $\approx 0,-6$ and $-10 \mathrm{~dB}$, respectively) is added to the transformed image data. For each image, 100 transformed and noisy images are made and classified with the RMS distance to the invariant features of the original images.

The correct classification ratios are shown in Table 2. More than $90 \%$ correct classification ratio is achieved for the randomly transformed images with the noise up to $-6 \mathrm{~dB}$. Note that the simple RMS distance to the original data is used here. It can be shown that the performance is still improved when optimization and learning techniques are applied.

Table2. Correct classification ratio for $2 \mathrm{D}$ texture images.

\begin{tabular}{cc} 
S.D. of noise (SNR (dB)) & correct classification ratio (\%) \\
\hline $0.0(\infty)$ & 100 \\
$40.0(0)$ & 94 \\
$80.0(-6)$ & 90 \\
$120.0(-9.5)$ & 67 \\
\hline
\end{tabular}




\subsection{D Brain Images}

The Normal Brain Database in the BrainWeb at McGill University [22] is used for 3D texture images. The database consists of the realistic MR images of the brain of $181 \times 217 \times 181$ voxels $(1 \mathrm{~mm}$ size), which are generated using the MRI simulator. Five $3 \mathrm{D}$ image data of $16 \times 16 \times 16$ voxels are taken from the brain image: (a) right anterior region, (b) right posterior region, (c) left anterior region, (d) left posterior region, (e) central region.

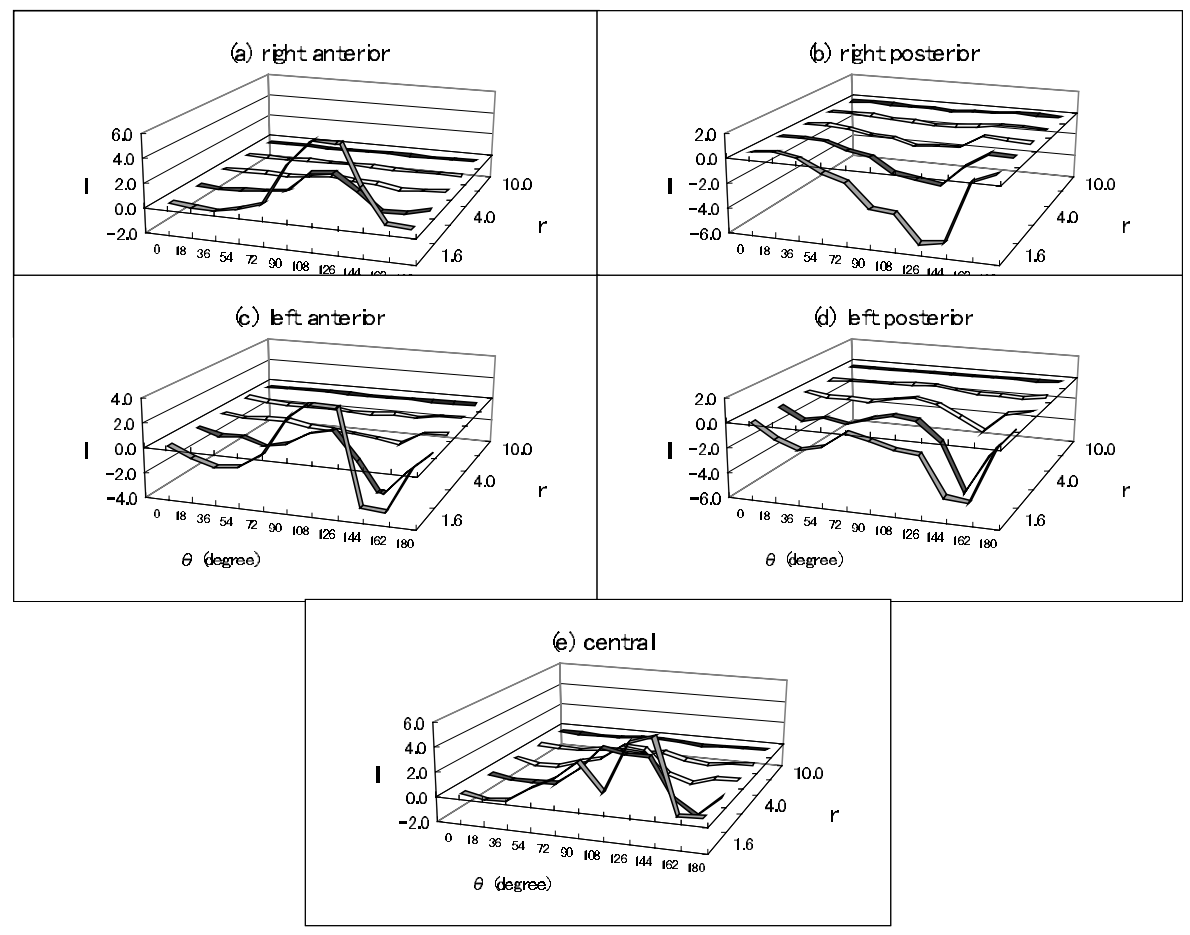

Fig. 2. Invariant feature $I(r, \theta)$ of $3 \mathrm{D}$ brain images

The invariant features $I(r, \theta)$ of the original images are shown in Fig. 2. Table 3 shows the RMS distances between the features of the original images and those of transformed and noisy images. The images are shifted from 0 to 0.5 by 0.1 along the $x$-axis and rotated from $0^{\circ}$ to $45^{\circ}$ by $5^{\circ}$ about the $x$-axis and by $15^{\circ}$ about the $y, z$-axes for each scaling factor. Those of 100 images with the white Gaussian noise $\mathrm{N}\left(0,25^{2}\right)$ (SNR is about $0 \mathrm{~dB}$ ) are also shown. Several transformed images magnified by double are not correctly classified with the RMS distance. The scaling gives variations in the invariant features larger than those in $2 \mathrm{D}$ images since the size of the 3D images are small.

Table 4 shows the correct classification ratios for randomly transformed and noisy images. The original image data are randomly shifted in $[-0.5,0.5]$ along the $x, y, z-$ axes and rotated in $\left[0^{\circ}, 360^{\circ}\right]$ about the three axes, with or without scaling by the 
factor in $[1.0,2.0]$ and adding the white Gaussian noise $\mathrm{N}\left(0,25^{2}\right)$. For each image, 100 image data are made and classified with the RMS distance of the invariant features. More than $80 \%$ correct classification ratio is achieved for the additive noise up to $0 \mathrm{~dB}$.

Table 3. RMS distance of the invariant feature of transformed 3D brain images.

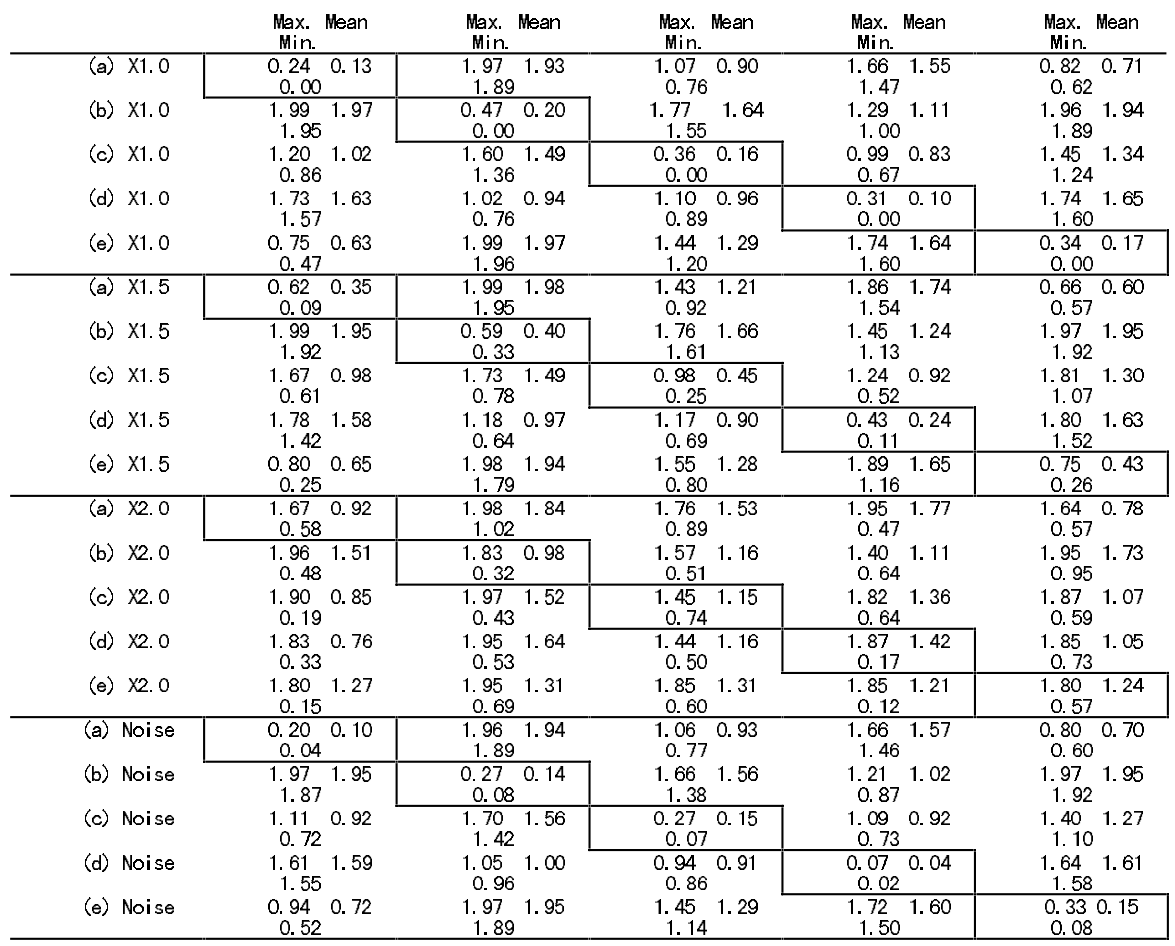

Table 4. Correct classification ratio for $3 \mathrm{D}$ brain images.

\begin{tabular}{ccc} 
S.D. of noise (SNR (dB)) & Scaling factor & $\begin{array}{c}\text { correct classification } \\
\text { ratio }(\%)\end{array}$ \\
\hline $0.0(\infty)$ & $\times 1.0$ & 98 \\
$25.0(0)$ & $\times 1.0$ & 99 \\
$0.0(\infty)$ & $\times 1.0 \sim \times 2.0$ & 87 \\
$25.0(0)$ & $\times 1.0 \sim \times 2.0$ & 86 \\
\hline
\end{tabular}

\section{Estimation of Rotation Angle}

The invariant feature is applied to the estimation of the rotation angles of $2 \mathrm{D}$ texture images.

First, we take a complex-valued function $f_{c}(x, y)$ obtained from the gradient field of images. 


$$
\begin{array}{r}
f_{c}(x, y)=f_{r}(x, y)+\mathrm{i} f_{i}(x, y), \quad f_{r}(x, y)=f(x, y)+f(x, y+1)-f(x+1, y)-f(x+1, y+1) \\
f_{i}(x, y)=f(x, y)-f(x, y+1)+f(x+1, y)-f(x+1, y+1)
\end{array}
$$

The image $f\left(x, y ; \theta_{t}\right)$ rotated by $\theta_{t}$ has the phase change by $\theta_{t}$.

$$
f_{c}\left(x, y ; \theta_{t}\right)=f_{c}(x, y ; 0) \exp \left(\mathrm{i} \theta_{t}\right)
$$

It can be shown that the phases of the bispectrum and the invariant feature $I(r, \theta)$ are also changed by $\theta_{t}$. The LMS estimate $\theta_{e}$ of the rotation angle $\theta_{t}$ of the image is given by

$$
\begin{gathered}
\theta_{e}=\operatorname{Tan}^{-1}\left\{\sum\left(-\operatorname{Re} I\left(\theta_{t}\right) \operatorname{Im} I(0)+\operatorname{Im} I\left(\theta_{t}\right) \operatorname{Re} I(0)\right)\right. \\
\left./ \sum\left(\operatorname{Re} I\left(\theta_{t}\right) \operatorname{Re} I(0)+\operatorname{Im} I\left(\theta_{t}\right) \operatorname{Im} I(0)\right)\right\} \\
\left(\left|I\left(r, \theta, \theta_{t}\right)-I(r, \theta ; 0) \exp \left(\mathrm{i} \theta_{e}\right)\right|^{2} \rightarrow \text { min. }\right)
\end{gathered}
$$

Figure 3 shows the LMS estimates for the rotation angles of the 2D texture images used in 3.1, where (a) without noise, (b) with $\mathrm{N}\left(0,20^{2}\right)$, (c) with $\mathrm{N}\left(0,40^{2}\right)$ and (d) scaled by 1.5. The mean and standard deviation of the estimation errors are shown in Table 5. The standard deviation of the estimation errors is less than $10^{\circ}$ for the noise up to $6 \mathrm{~dB}$ and for the scaling up to 1.5 .

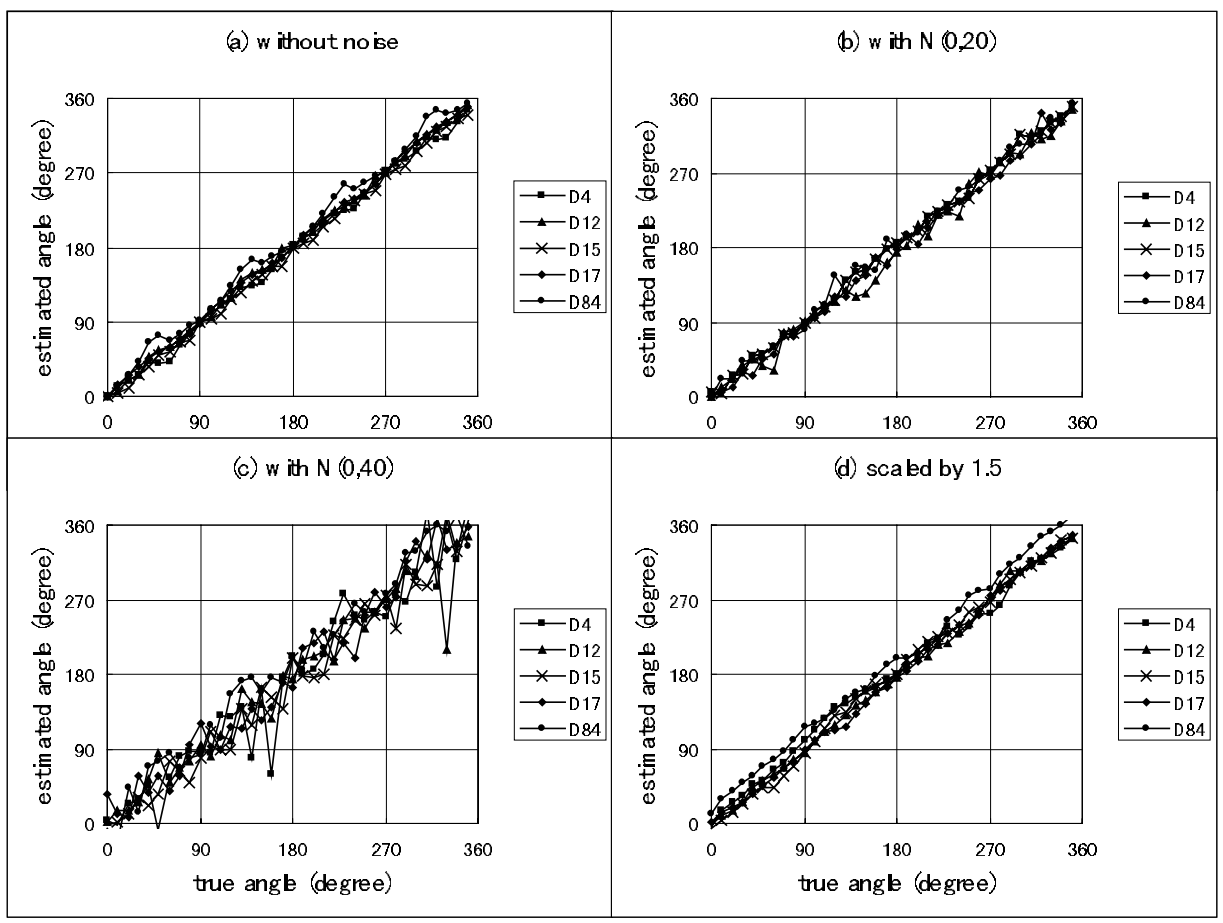

Fig. 3. Estimation of rotation angle of 2D texture images. 
Table 5. Mean and S.D. of the estimation errors of rotation angles.

\begin{tabular}{lccrc} 
& $\begin{array}{c}\text { S.D. of noise (SNR } \\
(\mathrm{dB}) \text { ) }\end{array}$ & Scaling factor & \multicolumn{3}{c}{ Mean and S. D. of estimation error } \\
(degree)
\end{tabular}

\section{Discussion}

The invariant feature based on the bispectrum is applied to the classification of 2D and 3D texture images suffering from rotation, scaling and additive noise. The high performance is obtained for arbitrary rotation, scaling up to double and additive noise up to $0 \mathrm{~dB}$. The feature is also invariant to linear changes in the gray-scale values of image data.

The RMS distance of the invariant features between the transformed images and the original ones are used and no a priori knowledge of the transformations is assumed in the classification experiment. The use of optimization techniques can increase the classification performance and make it possible to classify similar texture images the RMS distance of the features of which is more small.

The advantage of the features based on the third-order statistics is the robustness to noise. The invariant feature is effective even for the additive noise less than $0 \mathrm{~dB}$ SNR, where most approaches based on the second-order statistics are not applicable.

Computational complexity in the calculation of the bispectrum is avoided to some extent by using the voting method. The computational time is in $O\left(N^{2 d}\right)$ and the space is in $O\left(N^{d}\right)$, where $N^{d}$ is the number of pixels of image data ( $d$ : dimension). The calculation for one image data is done in several minutes with the SUN workstation in the experiment, which is in a range of practical use.

\section{References}

1. Tan, T. N.: Geometric transform invariant texture analysis. Proc. SPIE 2488 (1995) 475-485

2. Kashyap, R. L. and Khotanzaed, A.: A model-based method for rotation-invariant texture classification. IEEE Trans. PAMI. 8 (1986) 472-481

3. Cohen, F. S. et al.: Classification of rotated and scaled texture images using Gaussian Markov random field models. IEEE Trans. PAMI. 13 (1991) 192-202

4. Leung, M. M and Peterson, A. M.: Scale and rotation invariant texture classification. Proc. 26th Asilomar Conf. Signals, Systems and Computers 1 (1992) 461-465

5. You, J. and Cohen, H. A.: Classification and segmentation of rotated and scaled texture images using tuned masks. Pattern Recognition 26 (1993) 245-258

6. Chen, J.-L. and Kundu, A.: Rotational and gray-scale transform invariant texture identification using wavelet decomposition and hidden Markov models. IEEE Trans. PAMI 16 (1994) 208-214

7. Greenspan, H. et al.: Rotation invariant texture recognition using a steerable pyramid. Proc. ICPR'94 (1994) 162-167

8. Tan, T. N.: Noise robust and rotation invariant texture classification. Proc. 12th European Signal Processing Conf. (1994) 1377-1380 
9. Haley, G. M. and Manjunath, B. S.: Rotation invariant texture classification using modified Gabor filters. Proc. IEEE ICIP'95 (1995) 262-265

10. $\mathrm{Wu}, \mathrm{Y}$. and Yoshida, Y.: An efficient method for rotation and scaling invariant texture classification. Proc. ICASSP'95 4 (1995) 2519-2522

11. $\mathrm{Wu}, \mathrm{W} .-\mathrm{R}$. and Wei, S.-C.: Rotation and gray-scale transform-invariant texture classification using spiral resampling, subband decomposition, and hidden Markov model. IEEE Trans. Image Proc. 5 (1996) 1423-1434

12. Fountain, S. R. and Tan, T. N.: Extraction of noise robust and rotation invariant texture features via multichannel filtering. Proc. IEEE ICIP'97 3 (1997) 197-200

13. Fountain S. R. and Tan, T. N.: Efficient rotation invariant texture features for contentbased image retrieval. Pattern Recognition 31 (1998) 1725-1732

14. Manian, V. and Vasquez, R.: Scaled and rotated texture classification using a class of basis functions. Pattern Recognition 31 (1998) 1937-1948

15. Tan, T. N.: Rotation invariant texture features and their use in automatic script identification. IEEE Trans. PAMI 20 (1998) 751-756

16. Lohmann, A. W. and Wirnitzer, B.: Triple correlations. Proc. IEEE 72 (1984) 889901

17. Nikias, C. L. and M. R. Raghuveer, M. R.: Bispectrum estimation: a digital signal processing framework. Proc. IEEE 75 (1987) 869-891.

18. Tsatsanis, M. K. and Giannakis, G. B.: Object and texture classification using higher order statistics. IEEE Trans. PAMI. 14 (1992) 733-750

19. Murino, V. et al.: Noisy texture classification: a higher-order statistics approach. Pattern Recognition 31 (1998) 383-393

20. Hall, T. E. and Giannakis, G. B.: Bispectral analysis and model validation of texture images. IEEE Trans. Image Proc. 4 (1995) 996-1009

21. Brodatz, P.: Textures: A Photographic Album for Artists \& Designers. Dover, New York, (1966)

22. BrainWeb: Simulated Brain Database, http://www.bic.mni.mcgill.ca/brainweb/ 